\title{
Erratum to: Guide to Medical Image Analysis
}

Klaus D. Toennies

\section{Erratum to: \\ K.D. Toennies, Guide to Medical Image Analysis, Advances in Computer Vision and Pattern Recognition, DOI 10.1007/978-1-4471-7320-5}

In the original version of the book, in copyright page, the sentence "The author(s) has/have asserted their right(s) to be identified as the author(s) of this work in accordance with the Copyright, Design and Patents Act 1988." is to be added. The erratum book has been updated with the change.

The updated original online version of this book frontmatter can be found at http://dx.doi.org/10.1007/978-1-4471-7320-5 